\title{
Physarum Polycephalum changes polyaniline properties
}

\author{
Alice Dimonte ${ }^{1}$, Tatiana Berzina ${ }^{1}$ and Victor Erokhin ${ }^{1}$ \\ ${ }^{1}$ CNR-IMEM (National Council of the Researches - Institute of Materials for Electronics and Magnetism), Parco Area delle \\ Scienze 37A, 43124, Parma, Italy \\ alice.dimonte@imem.cnr.it
}

\begin{abstract}
Physarum polycephalum slime mould can modify polyaniline (PANI) features due to its internal activity. We created networks with different conductivity made by the slime mould on PANI substrates. Thus, Physarum's growth results in changing the conductivity state of PANI layers, providing negative and positive patterning of the samples. A spectrophotometric scanner is here exploited to investigate and characterize the effects coming out from the interaction between Physarum polycephalum and PANI. The latter is an electro-chromic polymer that vary its colour and conductive properties according to its redox state.
\end{abstract}

\section{Introduction}

Physarum polycephalum slime mould is attracting interest of scientists, not only biologists, because of its incredible features and adapting capabilities. Indeed, since many years, it has been the object of studies in the field of unconventional computing, (Gale et al. 2013), networks modelling and development (Nakagaki et al. 2004), biorobotics (Mayne et al. 2013), and biochemistry (Romeo et al. 2015). Physarum polycephalum slime mould is a single cell organism with many nuclei dispersed in the cytoplasm. It belongs to the family of Myxomycetes, Physarales species, taxonomically classified as Protozoas or, simply, slime mould (Ratzel et al. 2013). In nature it feeds on bacteria and decaying organic materials and needs darkness, humidity and a temperature around $25-27^{\circ} \mathrm{C}$. Therefore, the abovementioned characteristics have to be considered in order to properly culture a colony in a laboratory. Physarum polycephalum can be, thus, cultivated on agar gel or wet towels kept in a dark and humid chamber and fed with oatflakes. Moreover, to ensure its safety, the colony has to be periodically replanted to a new fresh substrate (agar or towels). Physarum polycephalum life is characterized by different phases (Stephenson et al. 2000): sporulation, sclerotium and plasmodium. The first is the reproduction phase, exploited by meiosis and fructification; the second is the dormant phase, a sort of hibernation during which the mould protect itself from not proper environmental conditions. The latter, plasmodium, is the vegetative form of slime mould; during this phase the organism is more active and moves searching for food. It appears as a yellowish multinuclear mass of protoplasm, a single cell with a myriad of nuclei, able to regenerate autonomously, even cutting a part away. Therefore, it has not a fixed mass, but it continuously changes its shape as a function of the spatial food distribution; indeed, it has been also demonstrated its capability of choosing not only the closest but also the most nutritional food sources from a buffet (Latty et al. 2009). Figure 1 shows an example of the abovementioned feature. Its foraging behaviour consists in the formation of optimized networks (Bonifaci et al. 2012) of protoplasmic tubes branching towards nutrients. However, in the case of food abundance it tends to stasis creating "pancakes-like" structures that enwrapped the whole food allowing phagocytosis (Golderer et al. 2001). These are astonishing features for a simple amoeba. In addition, as its name suggest, it produces a certain amount of slime, an extracellular polysaccharide (Simon et al. 1970) working as a sort of "external brain". The slime allows Physarum polycephalum to remember already trodden paths, in order to not retrace them when seeking for food or other attractants (Nakagaki et al. 2001). The protoplasmic veins of plasmodium's network consist in a two phases sol-gel medium. The external, more rigid, gel is the ectoplasm and contains the sol, more fluid endoplasm (Wohlfarth-Bottermann, 1974) which is transported by means of proteins present in the ectoplasm, the actomiosins. The latter, by contracting, generates high-pressure gradients that push the endoplasm forward allowing locomotion of the whole organism, in the so called shuttle streaming mechanism (Matsumoto et al. 2008). The way by which Physarum feels attractors and food in a certain direction, as well as the individuation of the forces involved in the locomotion of this organism, are still unknown. In this interesting scenario, we exploited Physarum's capability of creating networks (Shirikawa and Gunji, 2007) to pattern polyaniline (PANI) samples.

PANI is a redox electro-chromic polymer widely studied, also by authors for organic memristive device realizations (Erokhin and Fontana, 2011). Moreover, a spectrophotometer device has also been used to get evidence of the modifications induced by the mould on the PANI samples. In this work, we found a method by which it is possible to characterize Physarum's networks by electrical and spectrophotometric measurements,. The latter, built by the mould onto PANI substrate, were transferred, as a lithographic process, on PANI itself. 
Therefore, we finally project Physarum done networks on the PANI layer.

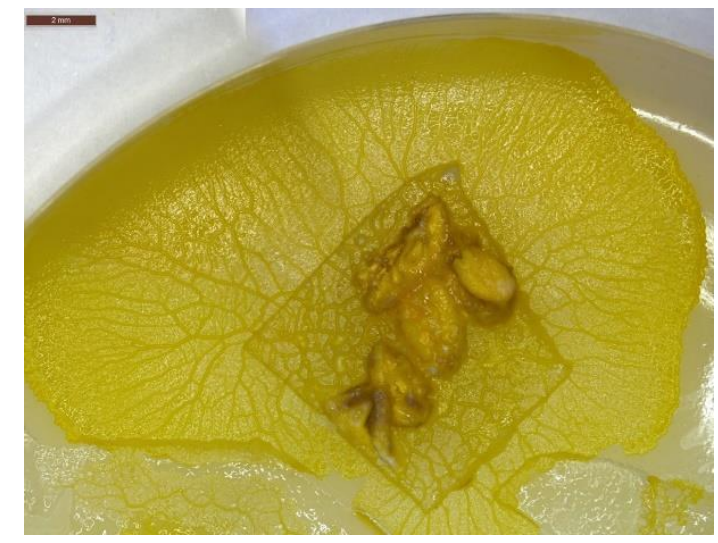

Figure 1: Optical microscope image of Physarum polycephalum on Agar nutrient gel, in this case it is clearly visible that the mould is creating a sort of "pancake-like" structure growing in all directions homogeneously. Thus demonstrating its shape is a function of spatial food distribution.

\section{Materials and methods}

\section{Physarum culture}

Physarum polycephalum slime mould, as mentioned in the introduction section, was cultured in dark and humid chamber at room temperature. The colony was maintained in Petri dishes with agar $1.5 \%$ non nutrient gel, fed with oat-flakes and periodically replanted to a new fresh agar.

\section{Spectrophotometer}

The device (Dimonte et al. 2015), a spectrophotometric scanner, is generally exploited in the art and restoration fields, being able to appreciate chromatic differences with high spatial resolution between points. The instrument produces an image consisting in an ordered collection of the spectral reflectance factors of each pixel.

The spectrophotometer here used is composed of a transmission spectrometer (Imspector V8 manufactured by Specim, Finland) designed for a $2 / 3$ inch CCD sensor equipped with a $25 \mu \mathrm{m}$ entrance slit and covering the $400 \div 780 \mathrm{~nm}$ spectral range with a spectral resolution of about $2 \mathrm{~nm}$. The spectrometer is coupled to a monochrome $2 / 3$ inch CCD matrix chill digital camera (Hamamatsu C4742-12bit, 1280×1024 pixels, $9 \mathrm{f} / \mathrm{sec}$ ) while a collecting lens (Computar TEC-M55 designed for a $2 / 3$ inch sensor) focalizes the painting on the plane of the entrance slit. The illumination is obtained by means of two $150 \mathrm{~W}$ halogen lamps whose light is filtered preventing the illumination of the painting. The digital camera is interfaced to a PC by means of a 12 bit frame grabber (Mutech MV1000). A software program drives the scanner, acquires data of a strip of the scene and allows to save its image as a spectral image. The program is implemented to reproduce the colours on a calibrated CRT monitor. The light is dispersed by the spectrometer and focalized in the plane, containing the sensor of the camera. The spectrometer has a 1:1 image magnification then, the image of the input is focalized on the pixel rows of the sensor, while its position along the vertical axis of the sensor, depends on the light wavelength. White light in the range $400 \div 780 \mathrm{~nm}$ enterig into the device fills the whole sensor. The acquisition of one frame of the digital camera can be thought as the acquisition of the reflectance spectra of each pixels of the strip of the painting.

\section{Polyaniline samples}

Polyaniline is a redox electrochromic polymer, discovered in 1985 (Kang et al. 1998). Its main feature is represented by a high conductivity difference between the conductive, green, oxidized state and the insulating, blue reduced one (Bredas et al. 1985). The transition depends on the doping degree.It is reversible and can be achieved by chemical treatment (i.e. water or basic solutions for reducing and acid chloride for oxidazing) or by proper voltage application (Berzina et al. 2007). Emeraldine base polyaniline was purchased from Sigma (Mn ca. 100,000). The deposition of the active PANI layer was carried out with a KSV 5000 LB trough, using a LangmuirSchaefer technique. Pure water, purified with a Milli-Q system, with a resistivity of $18.2 \mathrm{M} \Omega \mathrm{cm}$, serves as the subphase (Dimonte $e_{2}$ et al. 2014). Polyaniline powder was dissolved in 1methyl-2-pyrrolidonesNMPd and carefully filtered. The real concentration of the solution was determined and then NMP was added to achieve the final concentration of $0.2 \mathrm{mg} / \mathrm{ml}$.

\section{Oxygen-Containing Plasma Treatment}

The treatment has been performed with a Plasma Matrix bdiscom machine with a 10 min exposure at $99 \mathrm{~W}$. This allows us to remove PANI from the areas of the samples not covered by mould's network.

\section{Optical measurements}

The samples have been characterized by means of an optical microscope Leica D300. In addition, it was also fundamental, at the final state of the work, to make proper contacts of the PANI networks by means of micro-manipulating tips.

\section{Electrical characterizations}

The electrical conductivity of the PANI networks was recorded by means of a Keithley 2400 SourceMeters and two micromanipulators ending with tungsten tips.

\section{Experiments and Results}

Polyaniline samples were prepared by depositing 40 molecular layers of PANI on glass substrates by LangmiurShaefer technique (see material and methods); afterward the layer was doped by $\mathrm{HCl}$ treatment.

We put $2 \mu \mathrm{l}$ blob of Physarum polycephalum in the centre of a glass with deposited PANI film and attractors (oat flakes) at the external boundaries, to stimulate the mould in exploring the sample. Therefore, Physarum polycephalum, spanning towards the food sources in 8-12 hours, created networks on the polymer substrate as it is shown in Figure $2 a$ ). 
The sample was then transferred to open air and light conditions to let Physarum enter in the sclerotic phase and, consequently, maintain unvaried the designed network. Subsequently, we applied Oxygen plasma to remove PANI in all samples areas not covered by the mould.

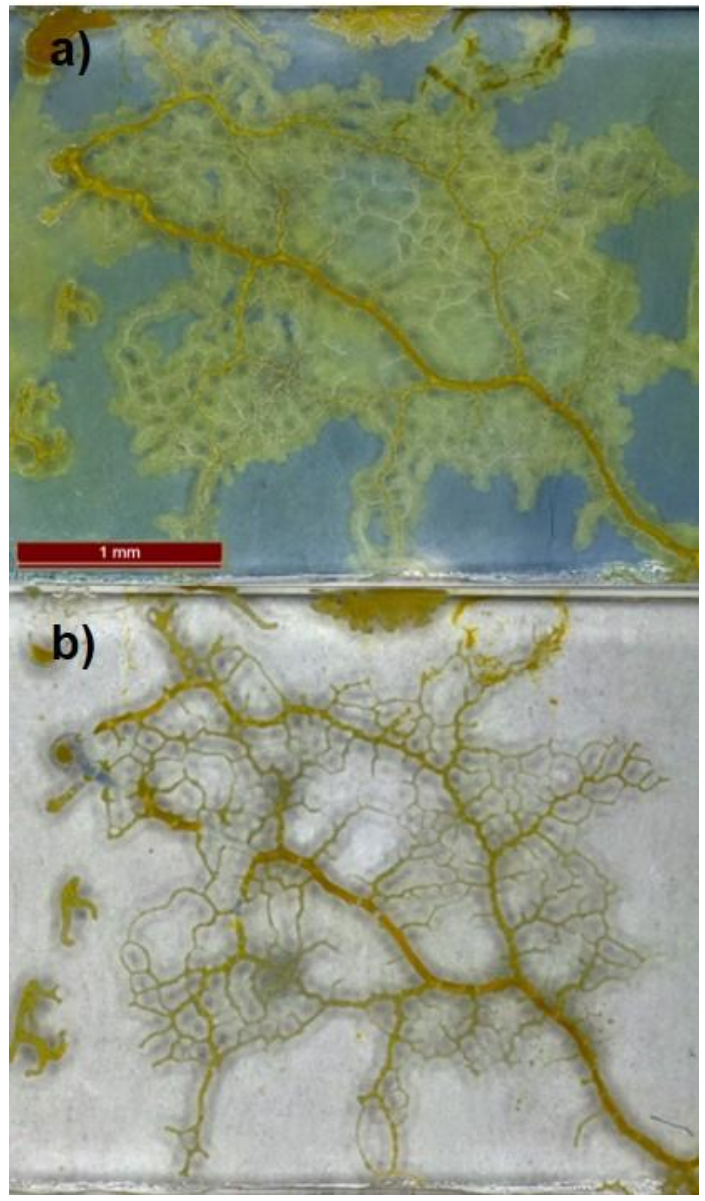

Figure 2 Optical microscope photograph of a network created by Physarum polycephalum on PANI sample before (part a) and after (part b) oxygen plasma treatment.
The result is shown in Figure 2b). The reported picture allows to conclude that the performed treatment did not destroy the network and the sclerotized mould stays attached to the surface without obvious damages. Successively, the mould was removed from the support surface by dipping the sample in water and than washing it gently. As expected, underneath the sclerotium we have found the PANI network. The PANI was doped to transfer it into conductive state by acid treatment (see materials and methods), we then contacted the channels of the network by means of micro-manipulating tips connected to a measurement station and verified that the most of channels were rather conductive with a calculated resistance in the range of 24-70 MOhm depending on the tested areas. Figure 3 shows the analysis scheme developed on the typical patterned samples. In particular it is clearly visible the green polyaniline network designed by Physarum.

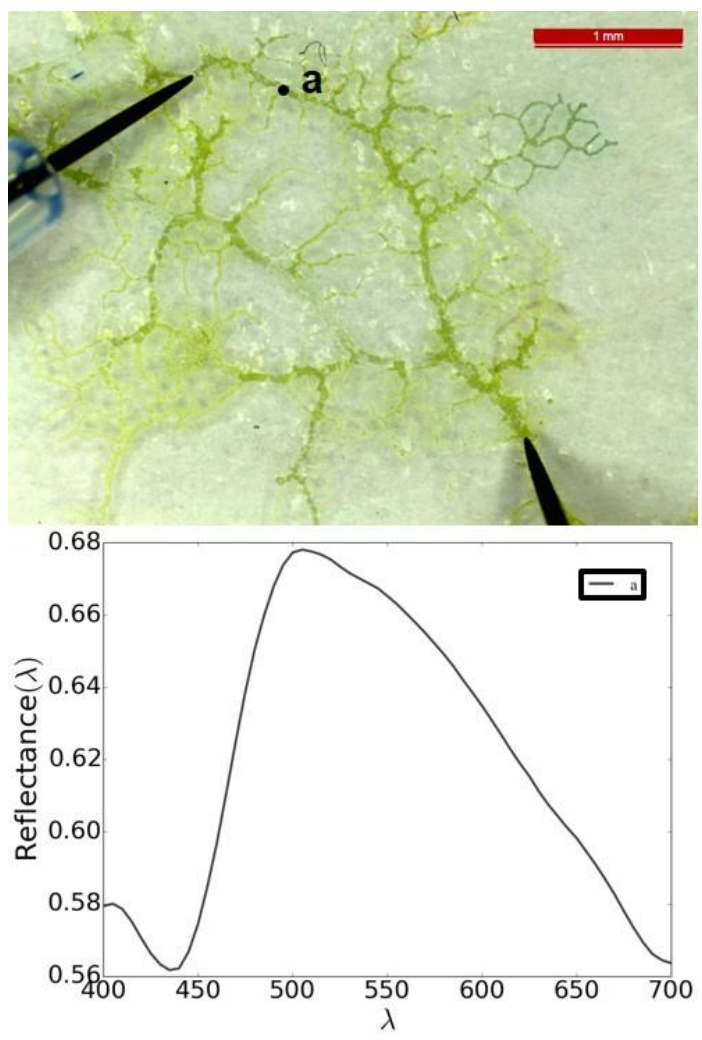

Figure 3: The part above shows an optical microscope photograph of an analyzed sample after Oxygen Plasma treatment and removal of the mold. The 2 black tips are the contact points for electrical characterization and the resulting value is $24 \mathrm{M} \Omega$. In the part below, the spectrum refers to the point " $a$ " signed in the image. Since the picture is a collection of spectra, it is possible to obtain spectra from lines or areas by selecting them directly on the image.

Alice Dimonte, Tatiana Berzina, Victor Erokhin (2015) Physarum Polycephalum changes polyaniline properties. Proceedings of the European Conference on Artificial Life 2015, pp. 14-17 
The latter can be considered as a type of lithography. Thw pattern, created during the slime mold growth can be transferred on the PANI layer, developed by the oxygen plasma and realizing the conductivity patterning after the acid treatment doping. Moreover, micromanipulating tips allow contacting the channels, one at a time, and checking the conductivity in desairable channels. The point marked on the image corresponds to the spectrum below. Indeed, after electrical characterizations, samples were measured with spectrophotometer and data have been elaborated considering pure polyaniline film as a reference.

\section{Conclusions}

The study here presented shows interesting results and opens many possible developments. Considering the noninvasiveness of the spectral analysis method (no influence on the conductivity variations), it will be possible to perform measurements in real time, characterizing the network during the motion of Physarum polycephalum. Therefore, we connected the growth of the slime mould with a variation of optical and electrical properties of the sub-layer (Dimonte et al. 2014). Here, putting a step forward, we demonstrated not only that Physarum polycephalum induces redox reactions, but also we exploited Physarum polycephalum's network as a lithographic mask. Therefore, we found and elaborated a technique by which it is possible to design conductive channels of polyaniline. Moreover, we applied the spectral imaging method for characterizing the patterned polyaniline surfaces formed during the Physarum polycephalum growth. Considering such kind of images, it is possible to appreciate chromatic differences with high spatial resolution between space-separated points. This is an important feature for the characterization of the grown networks of Physarum polycephalum. Therefore, the spectrophotometric scanner is here exploited in a non-conventional context, but it helps us to visualize the interactions between the growing mould and polyaniline layer.

Acknowledgments. This work is supported by the Future and Emerging Technologies (FET) programme within the Seventh Framework Programme for Research of the European Commission, under the Collaborative project PhyChip, grant agreement number 316366 .

\section{References}

Berzina, T., Erokhin, V. and Fontana, M. P. (2007) Spectroscopicinvestigation of an electrochemically controlled conducting polymer-solid electrolyte junction. J. Appl. Phys., 101: 024501 .

Bonifaci, V., Mehlhorn, K., Varma, G. (2012). Physarum can compute shortest paths. Journal of theoretical biology, 303:121-33.

Brédas, J. L. and Street, G. B. (1985). Polarons, bipolarons and solitons in conducting polymers. Acc. Chem. Res., 18: 309-315.

Dimonte, A., Berzina, T., Cifarelli, A., Chiesi, V., Albertini, F. and Erokhin, V. (2014). Conductivity patterning with Physarum polycephalum: natural growth and deflecting. Phys. Status Solidi $C$, 12:197-201

Dimonte $_{2}$, A., Pavesi, M., Berzina, T. and Erokhin, V. (2014). Hysteresis loop and cross-talk of organic memristive devices. Microelectronics Journal, 45(11):1396-1400.

Dimonte, A., Fermi, F., Berzina, T., Erokhin, V. (2015) Spectral imaging method for studying Physarum polycephalum growth on polyaniline surface. Materials Science and Engineering C, 53:11-14.

Erokhin, V., Berzina, T. and Fontana, M. P. (2005). A polymer based electrochemical device. J. Appl. Phys., 97: 064501.

Erokhin, V., Fontana, M. P. (2011). Thin film electrochemical memristive systems for bio-insoired computation. J. Computation and Theor. Nanosci, 8: 313-330

Gale, E., Adamatzky, A. and D. L. Costello, B. (2013). Are slime moulds living memristors? e-print arXiv:1306.3414.

Golderer, G., Werner, E., Leitner, S., Gröbner, P. and Werner-Felmayer, G. (2001). Nitric oxide synthase is induced in sporulation of Physarum polycephalum .Genes \& Dev.,15: 1299-1309

Kang, E., Neoh, K. and Tan, K. (1998). Polyaniline: a polymer with many intrinsic redox states. Progress in Polymer Science, 23: 277-324.

Latty, T. and Beekman, K. (2009). Food quality affects search strategy in the acellular slime mould, Physarum polycephlum. Behavioural Ecology, 20: 1160-1164.

Matsumoto, K., Takagi, S. and Nakagaki, T. (2008). Locomotive mechanism of Physarum polycephalum based on spatiotemporal analysis of protoplasmic streaming. Biophysical Journal, 94: 24922504.

Mayne,R., Patton, D., D. L. Costello, B. Patton, R. and Adamatzky, A. (2013). On loading slime mould Physarum polycephalum with metallic particles. Available at http://eprints.uwe.ac.uk/21054/

Nakagaki, T. (2001). Smart behavior of true slime mold in labyrinth. Res. Microbiol., 152: 767-770.

Nakagaki, T., Kobayashi, R., Nishiura, Y. and Ueda, T. (2004). Obtaining multiple separate food sources: behavioural intelligence in the Physarum polycephalum. Proc. R. Soc. Lond. B, 271-2305-2310.

Rätzel, V., Ebeling, B., Hoffmann, X., Tesmer, J. and Marwan, W. (2013). Physarum polycephalum mutants in the photocontrol of sporulation display altered patterns in the correlated expression of developmentally regulated genes. Dev. Growth Differ., 55(2) 247259.

Romeo, A., Dimonte, A., Tarabella, G., D’Angelo, P., Erokhin, V. and Iannotta S. (2015). A bio-inspired memory device based on interfacing Physarum polycephalum with an organic semiconductor. APL Materials, 3: 014909

Shirikawa, T. and Gunji, Y. (2007). Emergence of morphological order in the network formation of Physarum polycephalum. Biophys. Chem., 128:253-260.

Simon, H. L. and Henney, H. R. (1970). Chemical composition of slime from three species of myxomycetes. FEBS Letters, 70: 80-82.

Stephenson, S. L. and Stempen, H. Timber Press (2000). Myxomycetes: A. Handbook of Slime Molds. Portland, OR

Wohlfarth-Bottermann, K. E. (1974). Plasmalemma invaginations as characteristic constituents of plasmodia of Physarum polycephalum. J. Cell Sci. 16:23-32. 